\title{
Performance of Fuzzy Filter and Mean Filter for Removing Gaussian Noise
}

\author{
Pawan Kumar Patidar \\ Assistant Professor \\ Poornima College of Engineering, Jaipur, \\ Rajasthan, India \\ Rajasthan Technical University \\ Department of Computer Science \& Engineering
}

\author{
Pankaj Dadheech \\ Associate Professor \\ Swami Keshvanand Institute of Technology, \\ Management \& Gramothan, Jaipur, \\ Rajasthan,India \\ Rajasthan Technical University \\ Department of Computer Science \& Engineering
}

\begin{abstract}
The goal of image filtering is to remove the noise from the image in such a way that the "original" image is visible. Image filtering is a method by which we can enhance images .Image filtering methods are applied on images to remove the different types of noise that are either present in the image during capturing or injected into the image during transmission. Fuzzy Filter method for image de-noising based on Fuzzy set theory. This filter employs Fuzzy rules for deciding the gray level of a pixel within a window in the image. The Mean Filter is a linear filter which uses a mask over each pixel in the signal. Each of the components of the pixels which fall under the mask are averaged together to form a single pixel. This filter is also called as average filter. In these work Gaussian noise used and image filtering performed by fuzzy filter and mean filter. Further results have been compared for filters using Standard Deviation and Peak Signal to Noise Ratio.
\end{abstract}

\section{Keywords}

Gaussian noise, Fuzzy Filter, Mean Filter Standard Deviation, Peak Signal to Noise Ratio.

\section{INTRODUCTION}

Image Processing [2] is a form of signal processing for which the input is an image, such as a photograph or video frame;



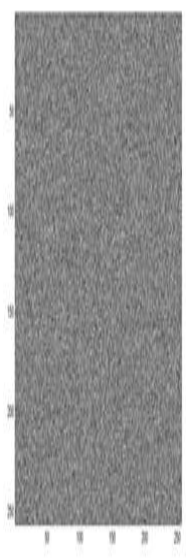

the output of image processing may be either an image or, a set of characteristics or parameters related to the image [3]. Linear filters generally blur sharp edges; destroy lines and other fine details present in the image. To overcome the problem of linear filtering, non-linear filtering [1] techniques become popular as an alternative to preserve signal structure. Mean filter is a linear filter and fuzzy filter is non linear filter. In this paper; we perform fuzzy and mean filtering to de-noise an image [4]. Fuzzy Filter can manage the imprecision and ambiguity in many image processing applications efficiently [5]. We suggest to de-noise a degraded image $\mathrm{X}$ given by $\mathrm{X}=$ $\mathrm{S}+\mathrm{N}$, where $\mathrm{S}$ is the original image and $\mathrm{N}$ is an Additive White Gaussian noise with unknown variance.

The rest of the paper is organized as follows:-

- In the second section we review the Fuzzy Filter.

- In the third section we present the method of Mean Filter.

- In the fourth section we described types of noise.

- The simulation results are discussed in part fifth.

- We conclude and future work in part sixth and seventh.

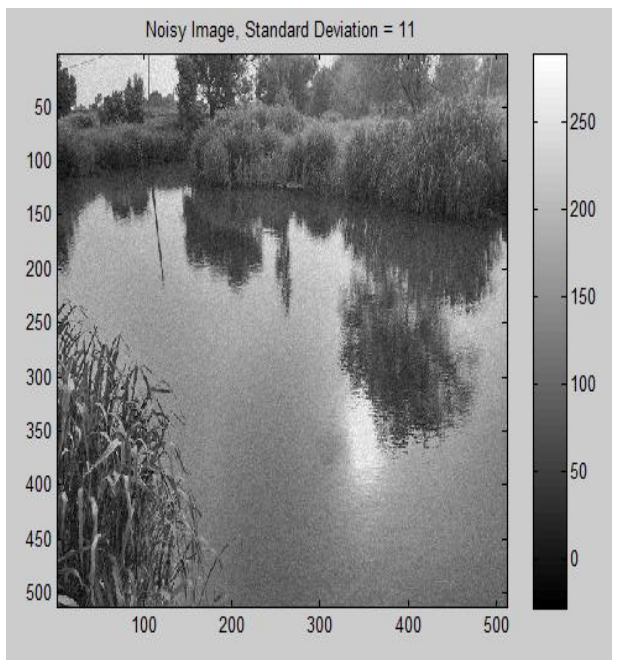

Fig. 1: The Original Image: An Additive White Gaussian Noise and Noisy Image

\section{FUZZY FILTER}

Fuzzy Filter (FF) is based on gray level mapping into a fuzzy plane, using a membership function [5]. The aim is to generate an image of higher contrast than the original image by giving a larger weight to the gray levels that are closer to the mean gray level of the image than to those that are farther from the mean. An image $f$ of size $\mathrm{Mx} \mathrm{N}$ and L gray levels can be considered as an array of fuzzy singletons, each having 
a value of membership denoting its degree of brightness relative to some brightness levels.

For an image $f(x, y)$, we can write in the notation of fuzzy sets:

$$
\begin{aligned}
\mathrm{f}(\mathrm{x}, \mathrm{y})= & \mathrm{U} \quad \mu_{\mathrm{xy}} / \mathrm{I}_{\mathrm{xy}} \\
\mathrm{x} \mathrm{y} & (2.1)
\end{aligned}
$$

where,

$$
\begin{aligned}
& x=1,2, \ldots \ldots, M \\
& y=1,2, \ldots \ldots, N \\
& I_{x y}=\text { The intensity of }(x, y) \text { th value } \\
& \mu_{x y}=\text { Membership value. }
\end{aligned}
$$

The membership function characterizes a suitable property of image like darkness, edginess, textural property etc. and can be defined globally for the whole image or locally for its segments. The basic principles of fuzzy enhancement scheme are illustrated in Fig. 1.

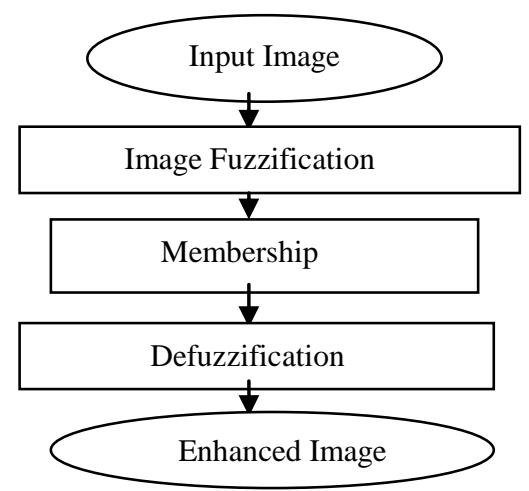

Fig. 1: The Basic Principles of Fuzzy Enhancement

Fuzzy Filter method for image de-noising based on Fuzzy set theory. This filter employs Fuzzy rules for deciding the gray level of a pixel within a window in the image. This is a variation of the MF and Neighborhood Averaging filter with fuzzy values.

The algorithm includes the following steps:

1. At first the gray values of the neighborhood $\operatorname{pixels}(n \times$ $n$ window) are stored in an array an thensorted in ascending or descending order.

2. Then, fuzzy membership value is assigned for eachneighbor pixels:

This step has the following characteristics:

i. A П-shaped membership function is used.

ii. The highest and lowest gray values get the membership value 0 .

iii. Membership value 1 is assigned tothe mean value of the gray levels of the Neighborhood pixels.

3. Now, we consider only $2 \times k+1$ pixels $(k / 2<=n 2)$ in the sorted pixels, and they are the median grayvalue and $\mathrm{k}$ previous and forward gray values in thesorted list.

4. Now, the gray value that has the highest membershipvalue will be selected and placed as output.

For example: Consider a 3x3 window of pixels as follows,

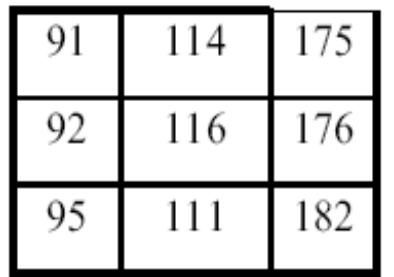

Here,

Original value: 116

Mean value: 128

Median value: 114

Let, Range value, $\mathrm{k}=2$

Sorted order: 91, 92, 95, 111, 114, 116, 175, 176, 182

Membership value: $0.0,0.0,0.03,0.56,0.69,0.76,0.04,0.03$, 0.00

Selected value is: 116

\section{MEAN FILTER}

We can use linear filtering to remove certain types of noise. Certain filters, such as averaging or Gaussian filters, are appropriate for this purpose. For example, an averaging filter is useful for removing grain noise from a photograph. Because each pixel gets set to the average of the pixels in its neighborhood, local variations caused by grain are reduced. Conventionally linear filtering Algorithms were applied for image processing. The fundamental and the simplest of these algorithms is the Mean Filter as defined in [4]. The Mean Filter is a linear filter which uses a mask over each pixel in the signal. Each of the components of the pixels which fall under the mask are averaged together to form a single pixel. This filter is also called as average filter. The Mean Filter is poor in edge preserving.

The Mean filter is defined by:

$$
\text { Mean filter }\left(\mathrm{x}_{1} \ldots . \mathrm{x}_{\mathrm{N}}\right)=\frac{1}{\mathrm{~N}} \sum_{\mathrm{i}=1}^{\mathrm{N}}
$$

where $\left(x_{l} \ldots \ldots x_{N}\right)$ is the image pixel range.

Generally linear filters are used for noise suppression

\section{IMAGE NOISE}

Image noise [6] is the random variation of brightness or color information in images produced by sensor and circuitry of scanner or camera.

The types of Noise are following:-

- Gaussian noise

- $\quad$ Salt-and-pepper noise

- $\quad$ Speckle noise

- Quantization noise

- $\quad$ Film grain

- Non-isotropic noise

\section{A. Gaussian Noise}

The standard model of amplifier noise is additive, Gaussian, independent at each pixel and independent of the signal intensity, caused primarily by thermal noise, including that which comes from the reset noise of capacitors ("kTC noise").Gaussian noise is a major part of the "read noise" of 
an image sensor, that is, of the constant noise level in dark areas of the image [6].

Gaussian noise is generated by formula given below:

Noise $=\mathrm{x} * \mathrm{y}$

Where $\mathrm{x}$ is standard deviation and $\mathrm{y}$ is randomly generated matrix of image size (M, M).Performances of different filters are tested for three different types of noise models by calculating the MSE and PSNR.

The values are calculated by the following expressions:

MSE is given by

M N

$M S E=\sum \sum(f(x, y)-k(x, y))^{2}$

$\mathrm{x}=1 \mathrm{y}=1$

Where $\mathrm{M}, \mathrm{N}$ is total number of pixels and $\mathrm{f}$ and $\mathrm{k}$ are original and filtered image respectively.

256

$\mathrm{PSNR}=20 \log _{10}$

Sqrt (MSE)

Where PSNR, MSE represents the peak signal to noise ratio and mean square error of the estimation.

\section{SIMULATION RESULTS}

In this section, experimental results are presented which explored the characteristics of the various filters used and tested. The comparative analysis has been presented on the basis of different standard deviation of noise for the original image $(512 * 512)$ which is shown in the Table (1). The result is taken by comparing the performance of Fuzzy Filter and Mean Filter on the basis of PSNR and MSE value.

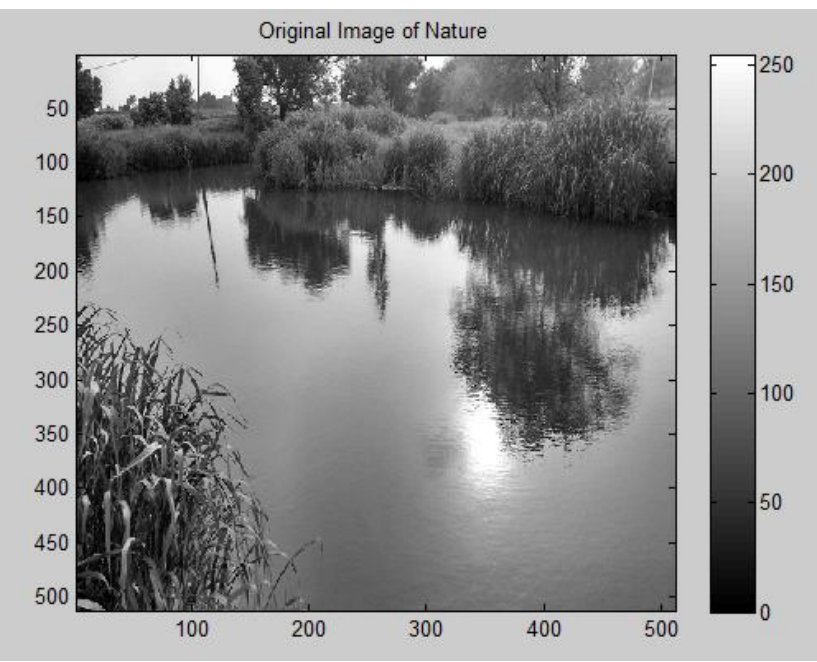

Fig. 1: Original Image



Fig. 2: Adding Gaussian Noise with Standard Deviation

(11)

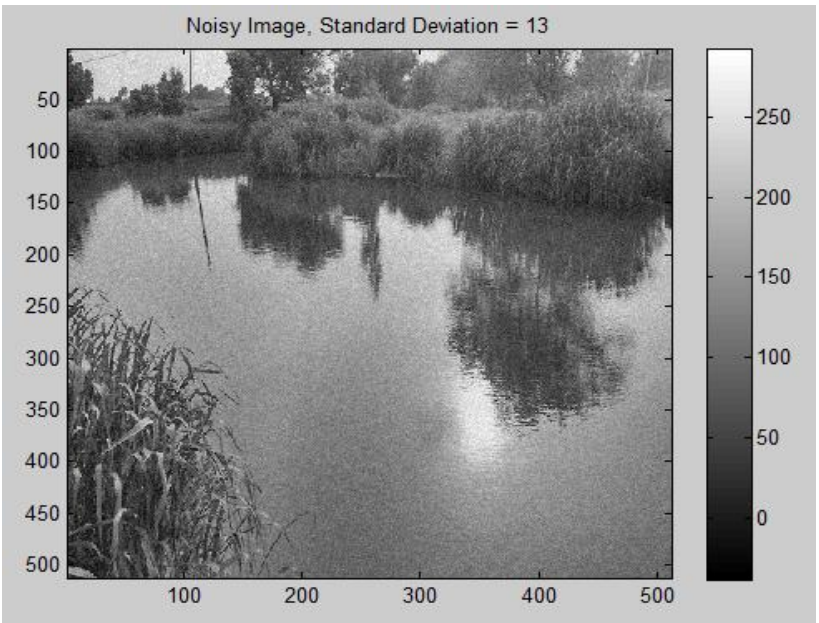

Fig. 3: Adding Gaussian Noise with Standard Deviation (13)

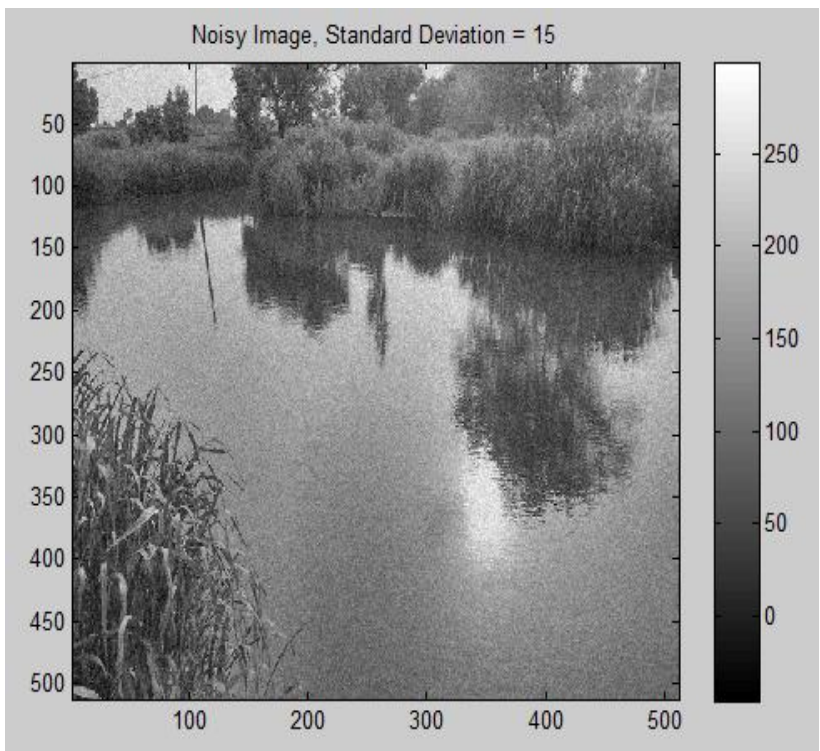

Fig. 4: Adding Gaussian Noise with Standard Deviation

(15) 


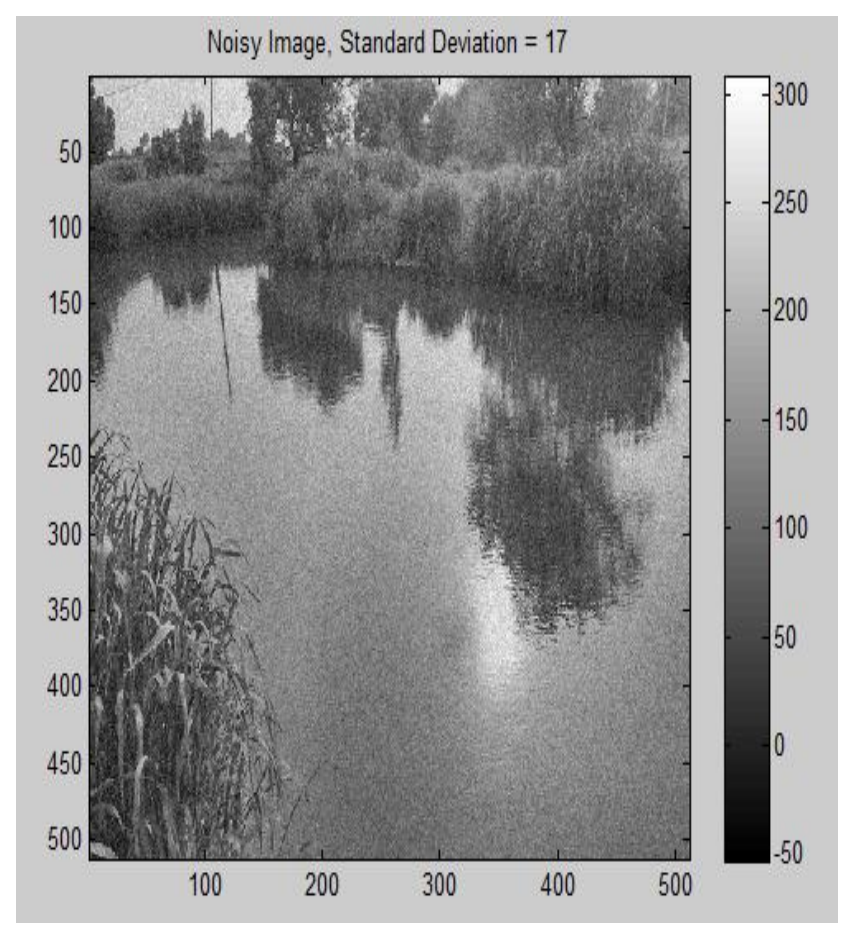

Fig. 5: Adding Gaussian noise with Standard Deviation

(17)

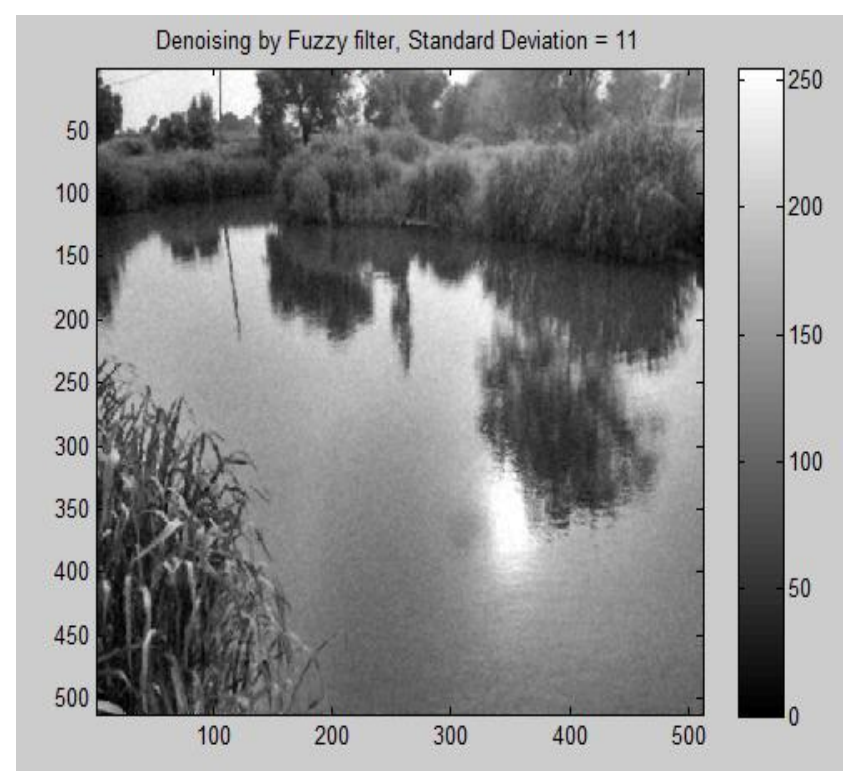

Fig. 6: De-noising by Fuzzy Filter, Standard Deviation =11



Fig. 7: De-noising by Fuzzy Filter, Standard Deviation =13

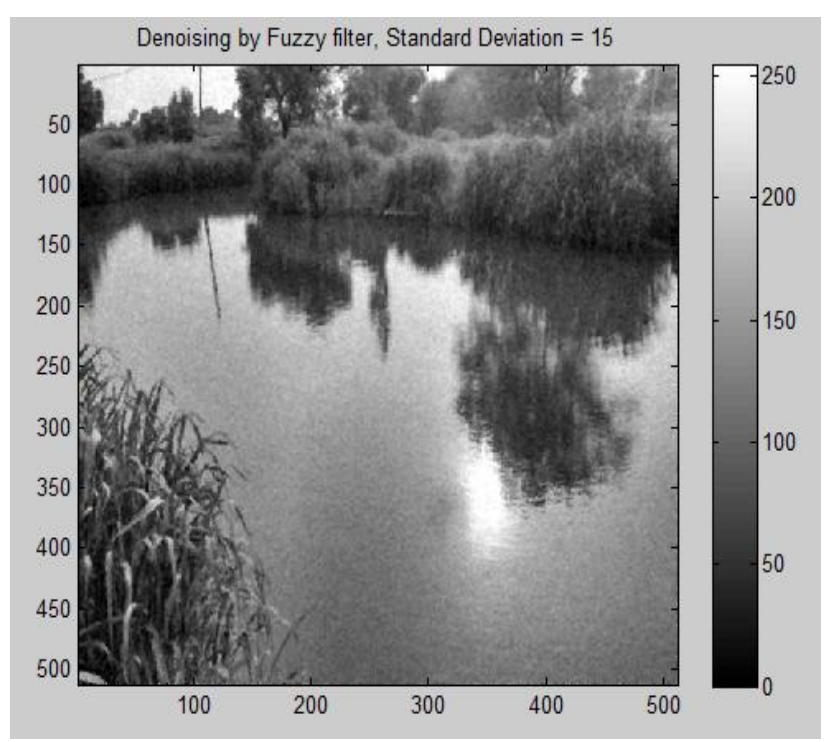

Fig. 8: De-noising by Fuzzy Filter, Standard Deviation $=15$ 


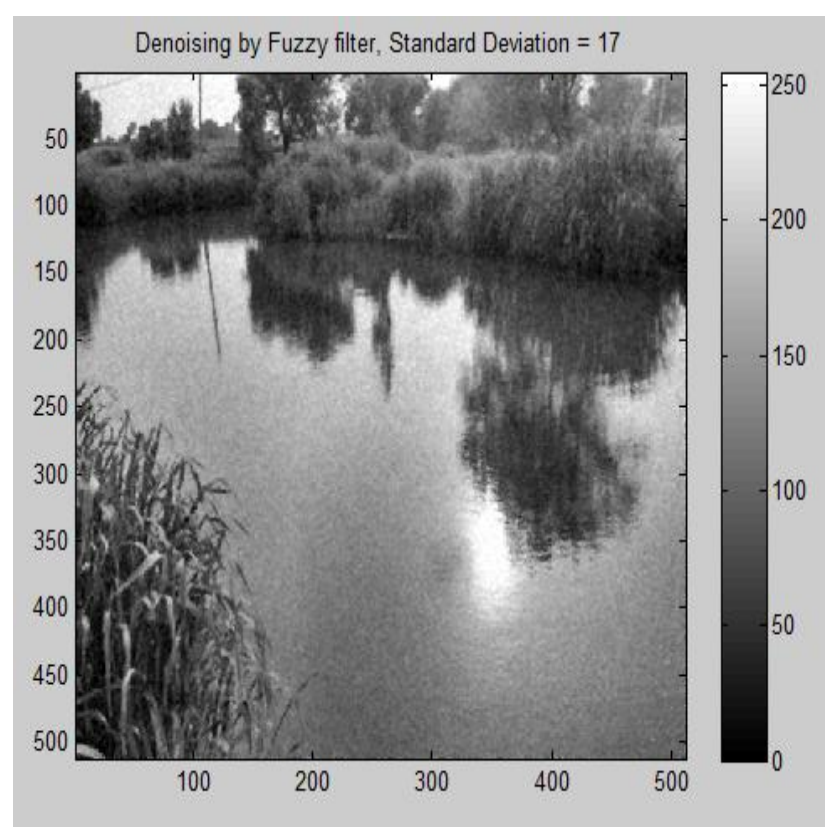

Fig. 9: De-noising by Fuzzy Filter, Standard Deviation $=17$

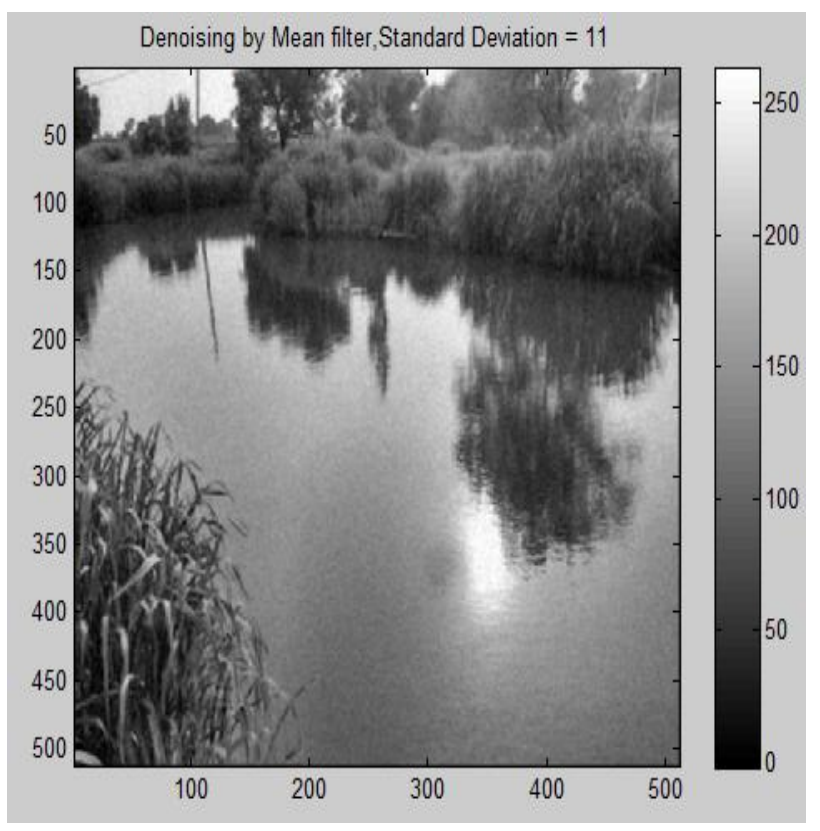

Fig. 10: De-noising by Mean Filter, Standard Deviation $=11$

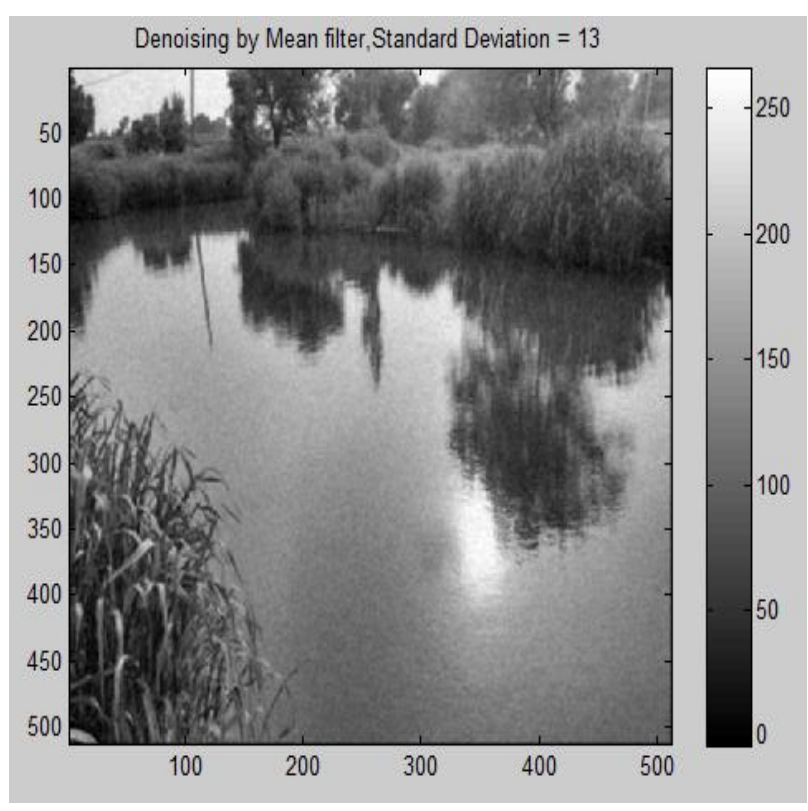

Fig. 11: De-noising by Mean Filter, Standard Deviation $=13$

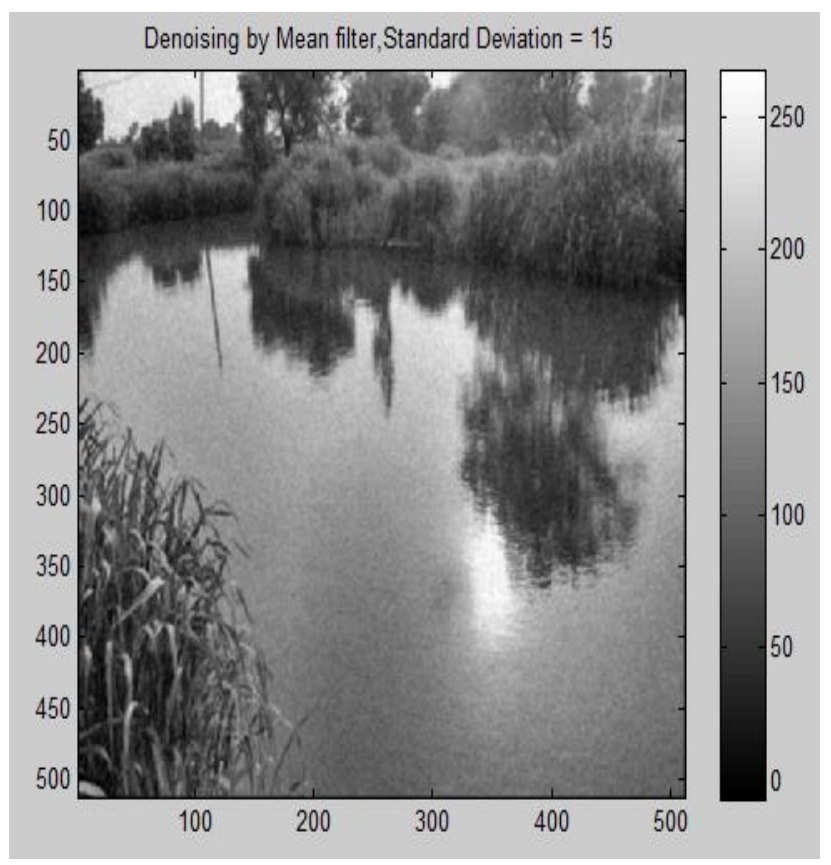

Fig. 12: De-noising by Mean Filter, Standard Deviation $=15$ 


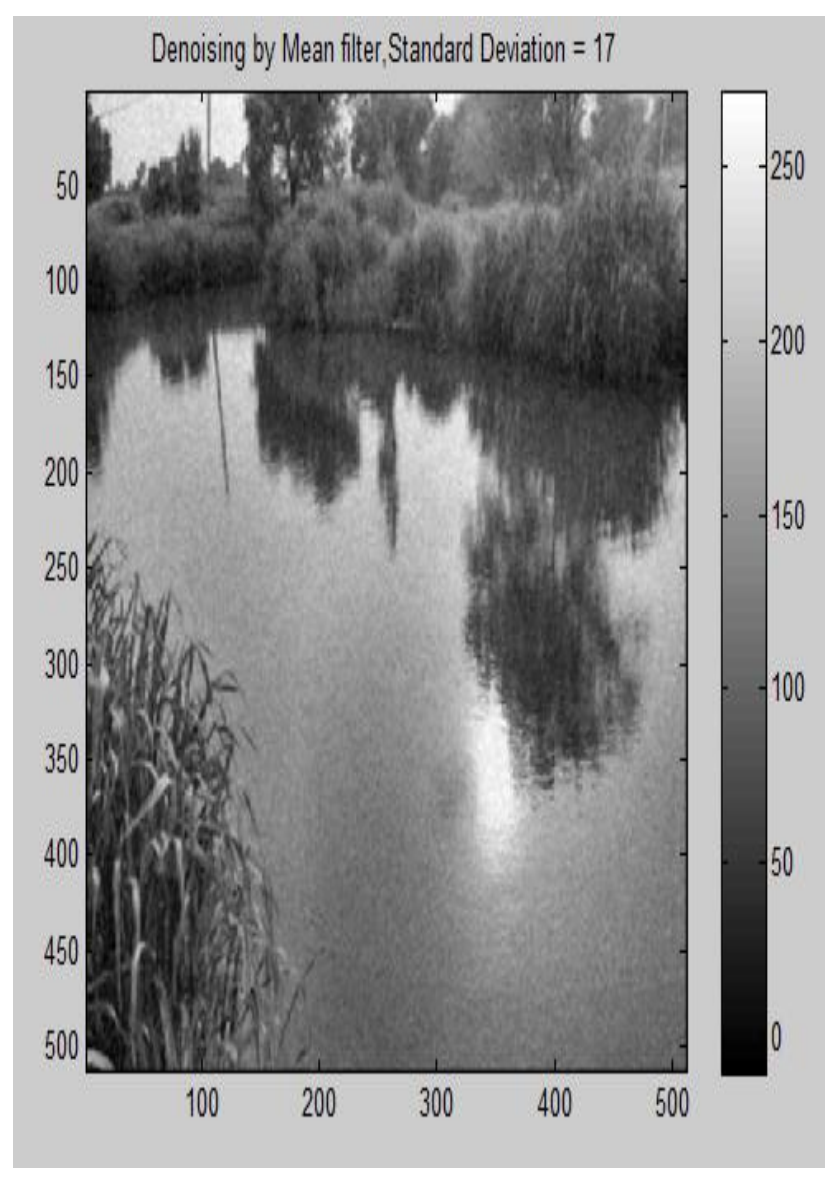

Fig. 13: De-Noising by Mean Filter, Standard Deviation $=17$

Table 1. Standard Deviation, MSE and PSNR values of Fuzzy Filter and Mean Filter

\begin{tabular}{|c|c|c|c|}
\hline \multirow{3}{*}{$\begin{array}{c}\text { Filter } \\
\text { Name }\end{array}$} & $\begin{array}{c}\text { Standard } \\
\text { Deviation }\end{array}$ & MSE Value & PSNR Value \\
\hline \multirow{3}{*}{$\begin{array}{c}\text { Fuzzy } \\
\text { Filter }\end{array}$} & 11 & 213.1429 & 24.8441 \\
\cline { 2 - 4 } & 13 & 220.3035 & 24.7006 \\
\cline { 2 - 4 } & 15 & 229.6217 & 24.5207 \\
\cline { 2 - 4 } & 17 & 239.2052 & 24.3431 \\
\hline \multirow{4}{*}{$\begin{array}{c}\text { Mean } \\
\text { Filter }\end{array}$} & 11 & 224.5876 & 24.6169 \\
\cline { 2 - 4 } & 13 & 229.8419 & 24.5165 \\
\cline { 2 - 4 } & 15 & 235.7155 & 24.4069 \\
\cline { 2 - 4 } & 17 & 242.5406 & 24.2830 \\
\hline
\end{tabular}



Fig. 14: Performance Measure of Fuzzy Filter and Mean Filter

\section{CONCLUSION}

This paper focus on the effective algorithms which have been used for Image Filtering by using fuzzy filter and mean filter which tell that: The performance of fuzzy filter is better than mean filter according to PSNR values for different standard deviation. PSNR high means good quality and low means bad quality. PSNR is using a term mean square error (MSE) in the denometer. So, low the error, high will be the PSNR. The performance measure of Fuzzy filter and Mean Filter is shown in Figure 14.

\section{SCOPE FOR FUTURE WORK}

Future works include use new concepts to modification of membership value without affecting the performance of result in Fuzzy filtering. Use adaptive properties for comparison of all filters. The construction of other fuzzy filtering methods for color and gray images to filtering other types of noise (salt $\&$ pepper, quantization, speckle, etc.).Use median or wiener filter for comparison with fuzzy filter for salt \& pepper noise, poisson noise or speckle noise. Use some new filters for comparison with different types of noise and different types of images (MRI Image, Mammogram Image ...etc.) which have different pixel value. Use new algorithms to cancellation noise from video frame. 


\section{REFERENCES}

[1] T. K. Thivakaran, Dr. R. M. Chandrasekaran, "Nonlinear Filter Based Image Denoising Using AMF Approach", International Journal of Computer Science and Information Security, Vol. 7, No. 2, 2010.

[2] Charu Khare, Kapil Kumar Nagwanshi, "Image RestorationTechnique with Non Linear Filter", International Journal ofAdvanced Science and Technology, Vol. 39, February, 2012.

[3] R.C. Gonzalez and R.E. Woods, Digital Image Processing, Prentice Hall, 2008.

[4] Mukesh C. Motwani, Mukesh C. Gadiya, Rakhi C.Motwani, Frederick C. Harris, Jr, (2004), "Survey of ImageDenoising Techniques," Proc. of GSPx, 2004, Santa Clara Convention Center, Santa Clara, CA, pp. 2730 .

[5] M. Mozammel Hoque Chowdhury, Md. Ezharul Islam,Nasima Begum and Md. Al-Amin Bhuiyan "Digital Image Enhancement with Fuzzy Rule-Based Filtering", IEEE, 1-4244-1551-9/07, 2007.

[6] Charles Boncelet (2005)."Image Noise Models" in Alan C. Bovik. Handbook of Image and Video Processing.

[7] S. A. Mahmood, "Fuzzy Enhancement for Color Image Processing," 2009 International Conference on Computer Technology and Development, Kota Kinabalu, 2009, pp. 340-344. doi: 10.1109/ICCTD.2009.229.

[8] A. S. Parihar, O. P. Verma and S. Tyagi, "An optimal fuzzy filter for Gaussian noise in color images using bacterial foraging algorithm," 2013 Annual International Conferenceon Emerging Research Areas and 2013 InternationalConference on Microelectronics, Communications andRenewable Energy, Kanjirapally, 2013, pp. 1-6.doi: 10.1109/AICERAICMiCR.2013.6576022.

[9] S. Chankhachon and S. Intajag, "Resourceful method to remove mixed Gaussian-Impulse noise in colorimages," 2015 12th International Joint Conference onComputer Science and Software Engineering
(JCSSE),Songkhla, 2015, pp. 18-23 doi: 10.1109/JCSSE.201.7219763.

[10] C. Wan, M. Ye, C. Yao and C. Wu, "Brain MR image segmentation based on Gaussian filtering and improvedFCM clustering algorithm," $2017 \quad 10^{\text {th }}$ International Congress on Image and Signal Processing, BioMedical Engineering and Informatics (CISP-BMEI), Shanghai,2017, pp. 1-5.doi: 10.1109/CISPBMEI.2017.8301978.

[11] YoungSik Choi and R. Krishnapuram, "Image enhancementbased on fuzzy logic,Proceedings" International Conference on Image Processing, Washington, DC, USA, 1995, pp.167-170 vol.1.doi: 10.1109/ICIP.1995.529066.

[12] F. Russo, "A technique for image restoration based on recursive processing and error correction", Proceedings ofthe $17^{\text {th }}$ IEEE Instrumentation and MeasurementTechnology Conference [Cat. No. 00CH37066], Baltimore,MD, USA, 2000, pp. 1232-1236 vol.3 doi:10.1109/IMTC.2000.848674.

[13] A. M. Joseph, M. G. John and A. S. Dhas, "Mammogram image denoising filters: A comparative study", 2017 Conference on Emerging Devices and Smart Systems (ICEDSS), Tiruchengode, 2017, pp. 184-189. doi: 10.1109/ICEDSS.2017.8073679.

[14] P. Dwivedy, A. Potnis, S. Soofi and P. Giri, "Performance comparison of various filters for removing different image noises", 2017 International Conference on Recent Innovations in Signal processing and Embedded Systems (RISE), Bhopal, 2017, pp. 181-186. doi: 10.1109/RISE.2017.8378150.

[15] Kshema, M. J. George and D. A. S. Dhas, "Preprocessing filters for mammogram images: A review," 2017 Conference on Emerging Devices and Smart Systems (ICEDSS), Tiruchengode, 2017, pp. 1-7. doi: 10.1109/ICEDSS.2017.8073694.

[16] X. Ji et al., "An effective self-adaptive mean filter for mixed noise", 2016 International Conference on Advanced Robotics and Mechatronics (ICARM), Macau, 2016, pp. 484-489.doi: 10.1109/ICARM.2016.7606968 